POS PROCEEDINGS

\title{
Comparison between LQCD and PNJL model at finite chemical potentials
}

\author{
Yuji Sakai ${ }^{* 1}$, Takahiro Sasaki ${ }^{1}$, Hiroaki Kouno ${ }^{2}$, Masanobu Yahiro ${ }^{1}$ \\ ${ }^{1}$ Department of Physics, Kyushu University, Fukuoka 812-8581, Japan \\ ${ }^{2}$ Department of Physics, Saga University, Saga 840-8502, Japan \\ ${ }^{*} E$-mail: sakaiephys.kyushu-u.ac.jp
}

Lattice QCD (LQCD) has the sign problem at real quark chemical potential. There are some regions with no sign problem; one is the imaginary quark chemical potential region and the others are the real and imaginary isospin chemical potential regions. We show that the Polyakov-loop extended Nambu-Jona-Lasinio (PNJL) model can reproduce LQCD data in the regions. We also determine the model parameters from the data and predict the QCD phase diagram in the real quark chemical potential region.

The XXVIII International Symposium on Lattice Field Theory, Lattice2010

June 14-19, 2010

Villasimius, Italy

\footnotetext{
*Speaker.
} 


\section{Introduction}

The QCD phase diagram is essential for understanding not only natural phenomena such as compact stars and the early universe but also laboratory experiments such as relativistic heavyion collisions. Quantitative calculations of the phase diagram from the first-principle lattice QCD (LQCD) have the sign problem at real quark chemical potential $\left(\mu_{\mathrm{q}}\right)$. Though several approaches have been proposed to circumvent the difficulty, these are still far from perfection.

As an approach complementary to the first-principle LQCD, we can consider effective models such as the Nambu-Jona-Lasinio (NJL) model and the Polyakov-loop extended Nambu-JonaLasinio (PNJL) model. The NJL model describes the chiral symmetry breaking, but not the confinement mechanism. The PNJL model is constructed so as to treat both the mechanisms. In the NJL-type models, the input parameters are determined at $\mu_{\mathrm{q}}=0$. It is then highly nontrivial whether the models predict the dynamics of QCD at finite $\mu_{\mathrm{q}}$ properly. This should be tested from QCD. Fortunately, this is possible in some regions without sign problem, such as imaginary $\mu_{\mathrm{q}}$, real and imaginary isospin chemical potential $\left(\mu_{\mathrm{I}}\right)$.

In this paper, we consider two-flavor QCD and show the reliability of the PNJL model by comparing the model result with LQCD data in their regions.

\section{Imaginary Quark Chemical Potential}

Roberge and Weiss [1] found that the thermodynamic potential, $\Omega_{\mathrm{QCD}}\left(\theta_{\mathrm{q}}\right)$, of QCD at imaginary chemical potential $\mu_{\mathrm{q}}=i \theta_{\mathrm{q}} T$ has a periodicity $\Omega_{\mathrm{QCD}}\left(\theta_{\mathrm{q}}\right)=\Omega_{\mathrm{QCD}}\left(\theta_{\mathrm{q}}+2 \pi k / 3\right)$, showing that $\Omega_{\mathrm{QCD}}\left(\theta_{\mathrm{q}}+2 \pi k / 3\right)$ is transformed into $\Omega_{\mathrm{QCD}}\left(\theta_{\mathrm{q}}\right)$ by the $\mathbb{Z}_{3}$ transformation with integer $k$. This means that QCD is invariant under a combination of the $\mathbb{Z}_{3}$ transformation and a parameter transformation $\theta_{\mathrm{q}} \rightarrow \theta_{\mathrm{q}}+2 \pi k / 3$. We call this combination the extended $\mathbb{Z}_{3}$ transformation. Thus, $\Omega_{\mathrm{QCD}}\left(\theta_{\mathrm{q}}\right)$ has the extended $\mathbb{Z}_{3}$ symmetry, and hence quantities invariant under the extended $\mathbb{Z}_{3}$ transformation have the RW periodicity [2].

We reveal that the PNJL model has the RW periodicity [2]. The two-flavor PNJL Lagrangian [3] in Euclidean spacetime is

$$
\mathscr{L}=\bar{q}\left(i \gamma_{v} D_{v}-\gamma_{4} \mu_{\mathrm{q}}+m_{0}\right) q-G_{\mathrm{s}}\left[(\bar{q} q)^{2}+\left(\bar{q} i \gamma_{5} \vec{\tau} q\right)^{2}\right]+U_{\Phi}\left(\Phi[A], \Phi^{*}[A], T\right),
$$

where $q$ denotes the two-flavor quark field, $m_{0}$ does the current quark mass, and $D_{v}=\partial_{v}-i A_{v} \delta_{v 0}$ with the gauge field $A_{v}$. In the chiral limit $\left(m_{0}=0\right)$, the Lagrangian density has the exact $S U(2)_{\mathrm{R}} \times$ $S U(2)_{\mathrm{L}} \times U(1)_{\mathrm{v}} \times S U(3)_{\mathrm{c}}$ symmetry. The Polyakov potential $U_{\Phi}$ [4] is a function of the Polyakov loop $\Phi=\frac{1}{3} \operatorname{tr}_{\mathrm{c}} L$ with $L=e^{i A_{4} / T}$ and its Hermitian conjugate $\Phi^{*}$. The PNJL thermodynamic potential $\Omega$ in the mean field approximation (MFA) is

$$
\Omega=-4 \int \frac{d^{3} \mathbf{p}}{(2 \pi)^{3}}\left[3 \varepsilon(\mathbf{p})+T \sum_{\lambda= \pm 1} \ln \operatorname{det}_{\mathrm{c}}\left(1+L^{\lambda} e^{-\varepsilon(\mathbf{p}) / T+i \lambda \theta_{\mathrm{q}}}\right)\right]+G_{\mathrm{s}} \sigma^{2}+U_{\Phi}
$$

where $\varepsilon=\sqrt{\mathbf{p}^{2}+M^{2}}, M=m_{0}-2 G_{\mathrm{s}} \sigma$, and $\sigma=\langle\bar{q} q\rangle$. The thermodynamic potential $\Omega$ is invariant under the extended $\mathbb{Z}_{3}$ transformation,

$$
L \rightarrow e^{-i 2 \pi k / 3} L, \quad \theta_{\mathrm{q}} \rightarrow \theta_{\mathrm{q}}+2 \pi k / 3 .
$$




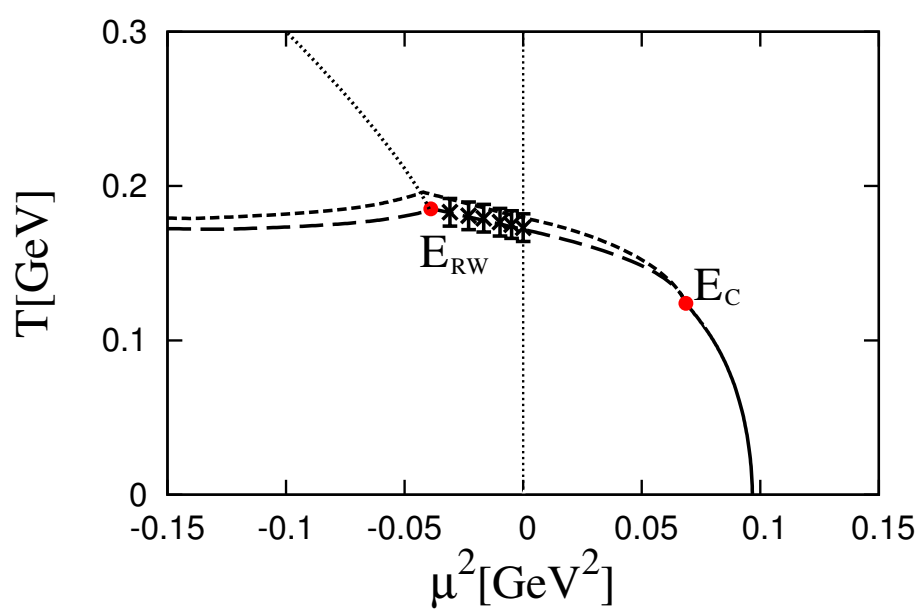

Figure 1: Phase diagram in the real and imaginary $\mu_{\mathrm{q}}$ regions by the PNJL model with the parameter set [2] that reproduces the LQCD data at imaginary $\mu_{\mathrm{q}}$. The points $\mathrm{E}_{\mathrm{RW}}$ and $\mathrm{E}_{\mathrm{C}}$ are the endpoints of the RW transition and the first-order chiral transition respectively. The solid line denotes the first-order chiral transition, the dashed (dotted) line does the crossover deconfinement (chiral) transition, and the dot-dashed line does the RW transition. Lattice data $(\times)$ are taken from [5].

Therefore, $\Omega$ has the RW periodicity.

At the present stage, the PNJL model is only a realistic effective model that possesses both the extended $\mathbb{Z}_{3}$ symmetry and the chiral symmetry [2]. This property guarantees that the phase diagram evaluated by the PNJL model has the RW periodicity in the imaginary $\mu_{\mathrm{q}}$ region, and therefore makes it possible to compare the PNJL result with LQCD data quantitatively in the imaginary $\mu_{\mathrm{q}}$ region. Actually, the PNJL model succeeds in reproducing the LQCD data «5] by introducing the vector-type four-quark interaction and the scalar-type eight-quark interaction [2]. The QCD phase diagram in the real $\mu_{\mathrm{q}}$ region is predicted by the PNJL model with the parameter set [2] that reproduces the LQCD data at imaginary $\mu_{\mathrm{q}}$, as shown in Fig. 1.
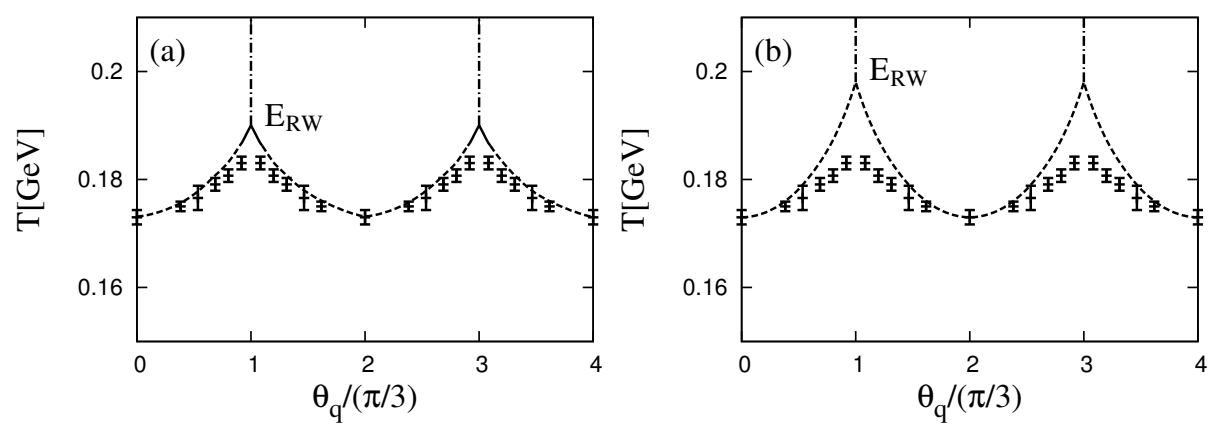

Figure 2: Phase diagrams of the deconfinement and the RW phase transition in the $\theta_{\mathrm{q}}-T$ plane with RRW-type $U_{\Phi}$ [4] (panel (a)) and F-type $U_{\Phi}$ [3] (panel (b)). The solid (dashed) line denotes the first-order (crossover) deconfinement transition, and the dot-dashed line does the RW transition. Point $\mathrm{E}_{\mathrm{RW}}$ is the endpoint of the RW transition. Lattice data (+) are taken from [5].

The phase diagrams of the deconfinement and the RW phase transition in the $\theta_{\mathrm{q}}-T$ plane by 
using the PNJL models with RRW-type $U_{\Phi}$ [4] and F-type $U_{\Phi}$ [3] are shown in Fig. 22 (a) and (b), respectively. Thus, the PNJL model with RRW-type $U_{\Phi}$ reproduces LQCD data [5] at finite $\theta_{\mathrm{q}}$, but the model with F-type $U_{\Phi}$ doesn't. In this sense, the PNJL model with RRW-type $U_{\Phi}$ calculation is more reliable.
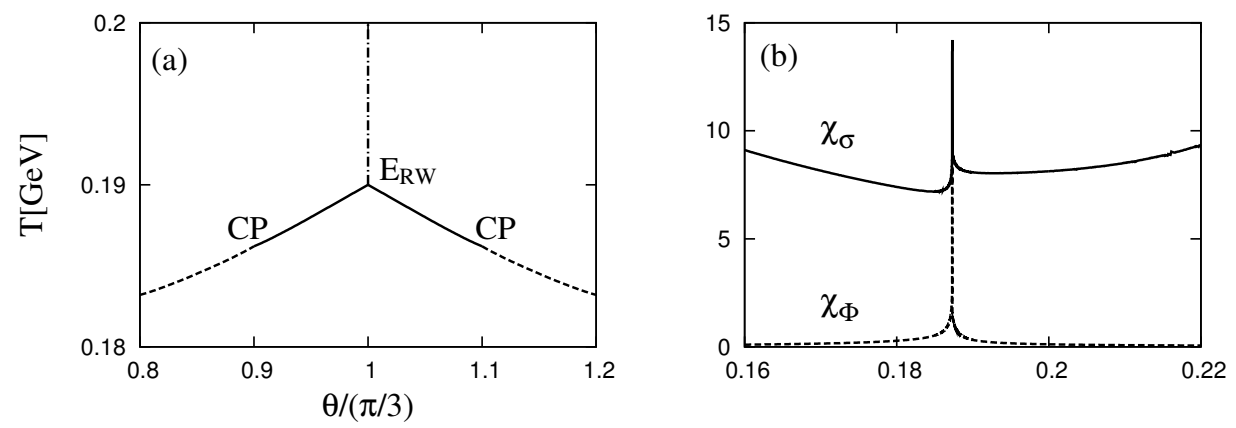

Figure 3: (a) The phase structure near $\mathrm{E}_{\mathrm{RW}}$ with RRW-type $U_{\Phi}$ is magnified. The solid (dashed) line denotes the first-order (crossover) deconfinement transition, and the dot-dashed line does the RW transition. Points $\mathrm{E}_{\mathrm{RW}}$ and $\mathrm{CP}$ are an endpoint of the RW transition and critical endpoints, respectively. (b) $T$ dependence of the chiral and Polyakov-loop susceptibilities, $\chi_{\sigma}$ and $\chi_{\Phi}$, at the point $\mathrm{CP}$.

The phase diagram for RRW-type $U_{\Phi}$ near $\mathrm{E}_{\mathrm{RW}}$ is magnified in Fig. 3(a). The RW endpoint is first order for RRW-type $U_{\Phi}$, but it's second order for F-type $U_{\Phi}[9]$. Thus, the order of the deconfinement phase transition near the RW endpoint strongly depends on $U_{\Phi}$ taken. The result of the PNJL calculation with RRW-type $U_{\Phi}$ is consistent with the LQCD data [6] where the order of the RW phase transition at $\mathrm{E}_{\mathrm{RW}}$ is first order for small quark mass. Point $\mathrm{E}_{\mathrm{RW}}$ is the triple point where the three first-order lines meet. Thus, there are two critical endpoints (CP) for each triple point ; $\mathrm{CP}$ is a point where the crossover and the first order lines meet. Figure 3 (b) shows the chiral and the Polyakov loop susceptibilities, $\chi_{\sigma}$ and $\chi_{\Phi}$, as a function of $T$ near CP. The susceptibilities are divergent at CP. Hence, the chiral and deconfinement transitions are second order at CP.

\section{Imaginary Isospin Chemical Potential}

LQCD has no sign problem at both real and imaginary $\mu_{\mathrm{I}}$. Recently, LQCD data were measured there and also in the case where both $\mu_{\mathrm{I}}$ and $\mu_{\mathrm{q}}$ are imaginary [7].

In the chiral limit, QCD has the chiral $S U_{\mathrm{L}}(2) \times S U_{\mathrm{R}}(2)$ symmetry when $\mu_{\mathrm{I}}=0$. However, at $\mu_{\mathrm{I}} \neq 0$ this symmetry is reduced to $U_{\mathrm{I}_{3}}(1) \times U_{\mathrm{AI}_{3}}(1)$, where $U_{\mathrm{I}_{3}}(1)$ is the isospin subgroup and $U_{\mathrm{AI}_{3}}(1)$ is the axial isospin subgroup. In the case $m_{u}=m_{d} \neq 0$, only the $U_{\mathrm{I}_{3}}(1)$ symmetry survives. When QCD vacuum keeps the $U_{\mathrm{v}}(1)$ and $U_{\mathrm{I}_{3}}(1)$ symmetries, the baryon charge $B=V\langle\hat{B}\rangle$ is either zero or integer and the isospin charge $I_{3}=V\left\langle\hat{I}_{3}\right\rangle$ is also either zero or half-integer, where $\hat{B}=$ $\bar{q} \gamma_{4} q, \hat{I}_{3}=\bar{q} \gamma_{4} I_{3} q$ and $V$ is the volume. In the partition function $Z$, the baryon- and the isospincharge operator appear through the form $\exp \left[V\left(2 i \theta_{\mathrm{I}} \hat{I}_{3}+i \theta_{\mathrm{q}} \hat{B}\right)\right]$ where $\mu_{\mathrm{q}, \mathrm{I}}=i T \theta_{\mathrm{q}, \mathrm{I}}$. Therefore, $Z$ has the periodicity $Z\left(\theta_{\mathrm{q}}, \theta_{\mathrm{I}}\right)=Z\left(\theta_{\mathrm{q}}, \theta_{\mathrm{I}}+2 \pi\right)$. In the isospin symmetric limit $m_{u}=m_{d}, Z$ is invariant under the interchange $u \leftrightarrow d$, i.e. $\theta_{\mathrm{I}} \rightarrow-\theta_{\mathrm{I}}$. Hence, $Z$ is invariant under charge conjugation, both $\theta_{\mathrm{q}} \rightarrow-\theta_{\mathrm{q}}$ and $\theta_{\mathrm{I}} \rightarrow-\theta_{\mathrm{I}}$. Furthermore we have proved that $Z$ has the RW periodicity at $\theta_{\mathrm{I}} \neq 0[9]$. 
All the relations are summarized as

$$
Z\left(\theta_{\mathrm{q}}, \theta_{\mathrm{I}}\right)=Z\left( \pm \theta_{\mathrm{q}}, \mp \theta_{\mathrm{I}}\right)=Z\left(\theta_{\mathrm{q}}, \theta_{\mathrm{I}}+2 \pi\right)=Z\left(\theta_{\mathrm{q}}+2 \pi / 3, \theta_{\mathrm{I}}\right) .
$$

Meanwhile, if the pion condensation occurs, the $U_{\mathrm{I}_{3}}(1)$ symmetry is spontaneously broken and the isospin charge is neither zero nor half-integer anymore. In this situation, QCD vacuum doesn't have the periodicities (3.1). We have proved that the pion condensation doesn't take place at imaginary $\mu_{\mathrm{I}}$ [9]. This can be understood intuitively. For real $\mu_{\mathrm{I}}$, the Bose-Einstein distribution function has an infrared divergence at $\mu_{\mathrm{I}} \geq m_{\pi} / 2$. This induces the Bose-Einstein Condensation, that is, the pion condensation. For imaginary $\mu_{\mathrm{I}}$, such a divergence never happens and hence no pion condensation occurs. As a result of this fact, $Z$ has all the discrete symmetries 3.1 .
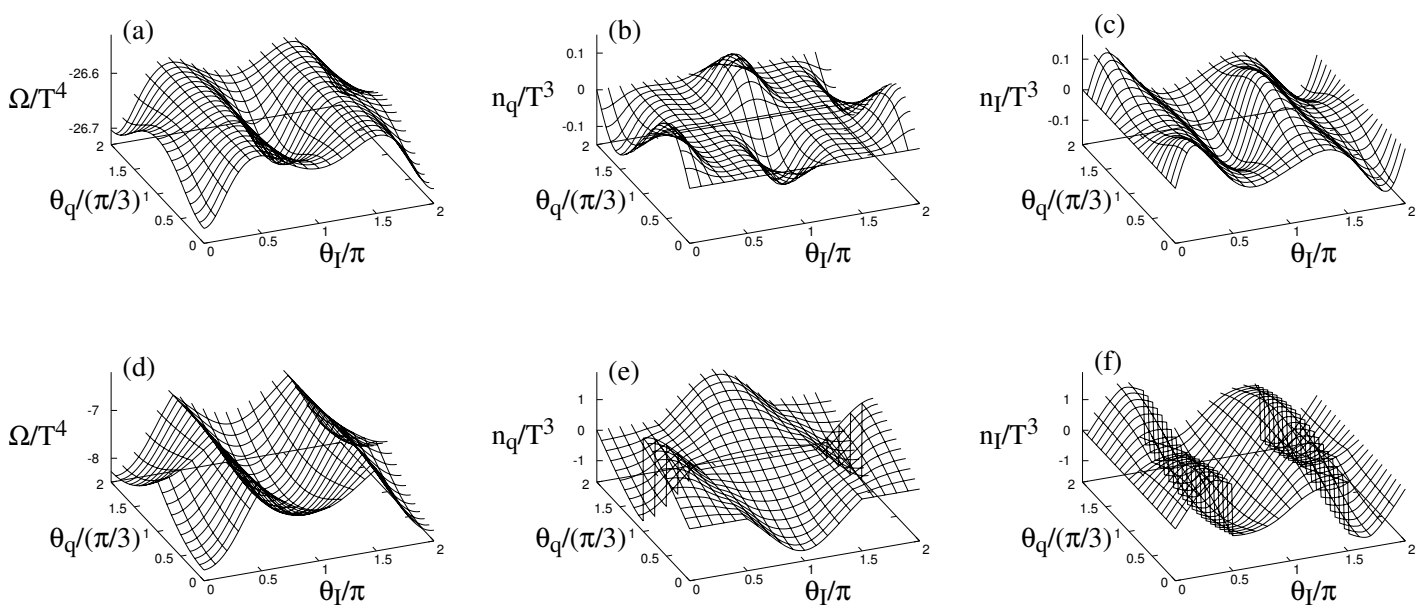

Figure 4: $\Omega / T^{4}, n_{\mathrm{q}} / T^{3}$ and $n_{\mathrm{I}} / T^{3}$ as a function of $\theta_{\mathrm{q}}$ and $\theta_{\mathrm{I}}$. Panels (a), (b) and (c) correspond to $T=$ $175 \mathrm{MeV}$, while panels (d), (e) and (f) to $T=250 \mathrm{MeV}$.

The absence of the pion condensation at imaginary $\mu_{\mathrm{I}}$ is true in the PNJL model [9]. The PNJL thermodynamic potential at $\mu_{\mathrm{I}} \neq 0$ in the MFA is

$$
\Omega=-2 \int \frac{d^{3} \mathbf{p}}{(2 \pi)^{3}} \sum_{f= \pm 1}\left[3 \varepsilon_{f}(\mathbf{p})+T \sum_{\lambda= \pm 1} \ln \operatorname{det}_{\mathrm{c}}\left(1+L^{\lambda} e^{-\varepsilon_{f}(\mathbf{p}) / T+i \lambda \theta_{\mathrm{q}}}\right)\right]+G_{\mathrm{s}}\left(\sigma^{2}+\pi^{2}\right)+U_{\Phi},(3.2)
$$

where $\varepsilon_{ \pm}=\sqrt{\left(\varepsilon(\mathbf{p}) \pm \mu_{\mathrm{I}}\right)^{2}+N^{2}}, N=2 G_{\mathrm{s}} \pi$. The pion condensate $\pi=\left\langle\bar{q} i \gamma_{5} \tau_{1} q\right\rangle$ is an order parameter of the spontaneous breakings of the $U_{\mathrm{I}_{3}}(1)$ symmetry. When there is no pion condensation, $\Omega$ is reduced to a simpler form

$$
\Omega=-2 \int \frac{d^{3} \mathbf{p}}{(2 \pi)^{3}}\left[6 \varepsilon(\mathbf{p})+T \sum_{\lambda, f= \pm 1} \ln \operatorname{det}_{\mathrm{c}}\left(1+L^{\lambda} e^{-\varepsilon(\mathbf{p}) / T+i \lambda \theta_{\mathrm{q}}+i f \theta_{\mathrm{I}}}\right)\right]+G_{\mathrm{s}} \sigma^{2}+U_{\Phi}
$$

which is invariant under the extended $\mathbb{Z}_{3}$ transformation $[2.3$, therefore $\Omega$ has the RW periodicity. The potential $\Omega$ has also the periodicity of $\theta_{\mathrm{I}} \rightarrow \theta_{\mathrm{I}}+2 \pi$. Furthermore $\Omega$ is invariant under the transformation, $\theta_{\mathrm{I}} \rightarrow-\theta_{\mathrm{I}}$, and also under the transformation, $\theta_{\mathrm{q}} \rightarrow-\theta_{\mathrm{q}}$ and $L^{ \pm} \rightarrow L^{\mp}$. These 
properties guarantee that the PNJL model possesses all the symmetries in 3.1, and the model reproduces LQCD data [7] qualitatively at imaginary $\mu_{\mathrm{I}}$ and $\mu_{\mathrm{q}}$.

Figure 4 shows $\Omega / T^{4}, \operatorname{Im}\left[n_{\mathrm{q}}\right] / T^{3}$ and $\operatorname{Im}\left[n_{\mathrm{I}}\right] / T^{3}$ as a function of $\theta_{\mathrm{q}}$ and $\theta_{\mathrm{I}}$ in the cases of $T=175$ and $250 \mathrm{MeV}$. Symmetries (3.1) are seen in Fig. (4) This result is consistent with LQCD ones [7]. If the pion condensate is nonzero, symmetries (3.1) break down. Hence, the fact that LQCD has symmetries (3.1) means that the pion condensation doesn't occur also in LQCD. As shown in Fig. 2 (a) for $\theta_{\mathrm{I}}=0$, at temperature above $T_{\mathrm{RW}}=190 \mathrm{MeV}$, there is the RW phase

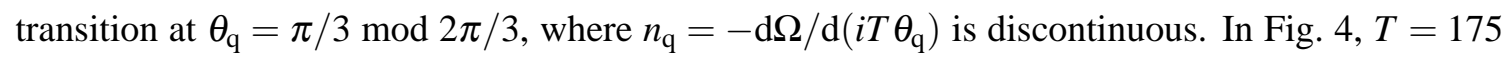
and $250 \mathrm{MeV}$ are typical temperatures below and above $T_{\mathrm{RW}}$, respectively. For any temperature, the RW periodicity is seen. Below $T_{\mathrm{RW}}$, these quantities are smooth at any $\theta_{\mathrm{q}}$ and $\theta_{\mathrm{I}}$. In contrast, above $T_{\mathrm{RW}}, \Omega$ and $n_{\mathrm{I}}$ have cusps at $\theta_{\mathrm{q}}=\pi / 3 \bmod 2 \pi / 3$, while $n_{\mathrm{q}}$ is discontinuous there. The discontinuity means the RW phase transition. Eventually, the transition occurs at $\theta_{\mathrm{q}}=\pi / 3 \mathrm{mod}$ $2 \pi / 3$ when $0 \leq \theta_{\mathrm{I}}<\pi / 2$ and $\pi<\theta_{\mathrm{I}} \leq 2 \pi$, and at $\theta_{\mathrm{q}}=0 \bmod 2 \pi / 3$ when $\pi / 2 \leq \theta_{\mathrm{I}} \leq 3 \pi / 2$ [.
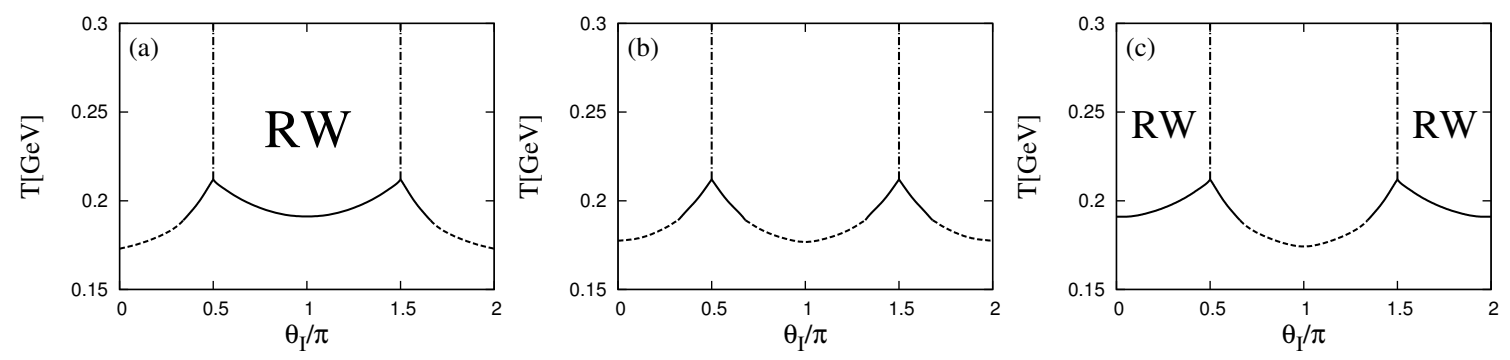

Figure 5: Phase diagram of the deconfinement phase transition in the $\theta_{\mathrm{I}}-T$ plane. Panels (a), (b) and (c) correspond to $\theta_{\mathrm{q}}=0, \pi / 6$ and $\pi / 3$, respectively. The solid (dashed) line denotes the first-order (crossover) transition. The area labeled by 'RW' between the two dot-dashed lines represents the region where the RW phase transition occurs.

Figure 5 shows the phase diagram of the deconfinement phase transition in the $\theta_{\mathrm{I}}-T$ plane. Near $\theta_{\mathrm{I}}=\pi / 2 \bmod \pi$, the deconfinement phase transition is first order in all panel (a)-(c). Near $\theta_{\mathrm{I}}=\pi \bmod \pi$, the deconfinement phase transition is first order at $\theta_{\mathrm{q}}=0$, but crossover at $\theta_{\mathrm{q}}=\pi / 6$ and $\pi / 3$. The RW phase transition occurs in the area labeled by 'RW' between the two dot-dashed lines.

Quantitative comparison of the PNJL model with LQCD data [7] is made at $T \leq T_{\mathrm{c}}$ by using the hadron resonance gas (HRG) model that can reproduce the LQCD data there. We have shown [9] that the PNJL model reproduces the LQCD data for the oscillatory patterns. For the magnitudes, meanwhile, the PNJL model underestimates the LQCD result. This discrepancy is understandable as follows. Below $T_{\mathrm{c}}$, hadronic excitations are important, but such an effect is not included in the MFA. By adding the hadronic correction to the PNJL model, the model agrees with the LQCD [9]. The HRG model works well at $T<T_{\mathrm{c}}$, but not at $T>T_{\mathrm{c}}$; especially the HRG model doesn't reproduce the RW phase transition. In contrast, the PNJL model with the hadronic correction works both below and above $T_{\mathrm{c}}$.

\section{Real Isospin Chemical Potential}

LQCD data are available at real $\mu_{\mathrm{I}}$ and $\mu_{\mathrm{q}}=0$ [8]. The scalar-type eight-quark interaction is 
necessary to reproduce LQCD data at imaginary $\mu_{\mathrm{q}}$ [2]. Figure 4(a) shows the phase diagram of the PNJL model with the scalar-type eight-quark interaction in the $\mu_{\mathrm{I}}-T$ plane at $\mu_{\mathrm{q}}=0$. The PNJL model with the eight-quark interaction is also consistent with the LQCD at $\mu_{\mathrm{I}} \neq 0$ [10]. There is a tricritical point (TCP) where the first-order pion-superfluidity phase transition line is connected to the second-order phase transition. The critical points such as CEP and TCP are important as indicators of the chiral and pion-superfluidity phase transitions at compact stars and laboratory experiments where $\mu_{\mathrm{I}}$ is nonzero generally. The TCP in the $\mu_{\mathrm{I}}-T$ plane at $\mu_{\mathrm{q}}=0$ is connected to the CEP in the $\mu_{\mathrm{q}}-T$ plane at $\mu_{\mathrm{I}}=0$ in the $\mu_{\mathrm{q}}-\mu_{\mathrm{I}}-T$ space [10], as shown in Fig. 6](b).
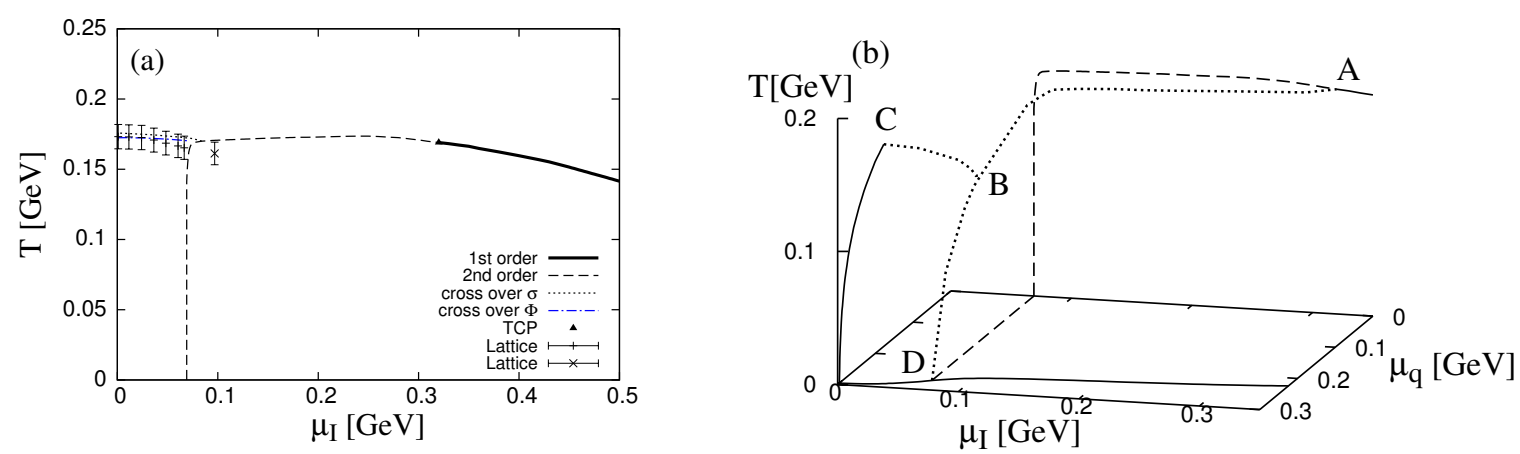

Figure 6: (a) Phase diagram in the $\theta_{\mathrm{I}}-T$ plane at $\theta_{\mathrm{q}}=0$ with the eight-quark interaction. The thicksolid (dashed) line denotes a first-order (second-order) pion-superfluidity phase transition. The dot-dashed (dotted) line denotes a deconfinement (chiral) crossover transition. Lattice data are taken from [8]. (b) Phase diagram in the $\mu_{\mathrm{I}}-\mu_{\mathrm{q}}-T$ space with the eight-quark interaction. Line ABC denotes the chiral CEP, ABD line does the pion-superfluid TCP. The CEP and the TCP coexist on line AB. The solid (dashed) line denotes the first (second) order transition.

\section{References}

[1] A. Roberge and N. Weiss, Nucl. Phys. B275, 734 (1986).

[2] Y. Sakai, et al., Phys. Rev. D 77, 051901 (2008); Phys. Rev. D 78, 036001 (2008); Phys. Rev. D 78, 076007 (2008); Phys. Rev. D 79, 096001 (2009).

[3] K. Fukushima, Phys. Lett. B 591, 277 (2004); Phys. Rev. D 77, 114028 (2008).

[4] S. Rößner, C. Ratti, and W. Weise, Phys. Rev. D 75, 034007 (2007).

[5] P. de Forcrand and O. Philipsen, Nucl. Phys. B642, 290 (2002).

[6] M. D’Elia and F. Sanfilippo, Phys. Rev. D 80, 111501 (2010).

[7] M. D’Elia and F. Sanfilippo, Phys. Rev. D 80, 014502 (2009).

[8] J. B. Kogut and D. K. Sinclair, Phys. Rev. D 70, 094501 (2004).

[9] Y. Sakai, H. Kouno, and M. Yahiro, J. Phys. G: Nucl. Part. Phys. 36, 115010 (2009).

[10] T. Sasaki, Y. Sakai, H. Kouno, and M. Yahiro, arXiv:hep-ph/1005.0910 (2010). 